Document downloaded from:

http://hdl.handle.net/10251/181088

This paper must be cited as:

Ortigosa, N.; Cano, O.; Mainardi, L. (2019). Distance and Similarity Measurements of P Waves Before and After Pulmonary Vein Isolation in Patients with Atrial Fibrillation. IEEE. 87-90. https://doi.org/10.1109/EMBC.2019.8857563

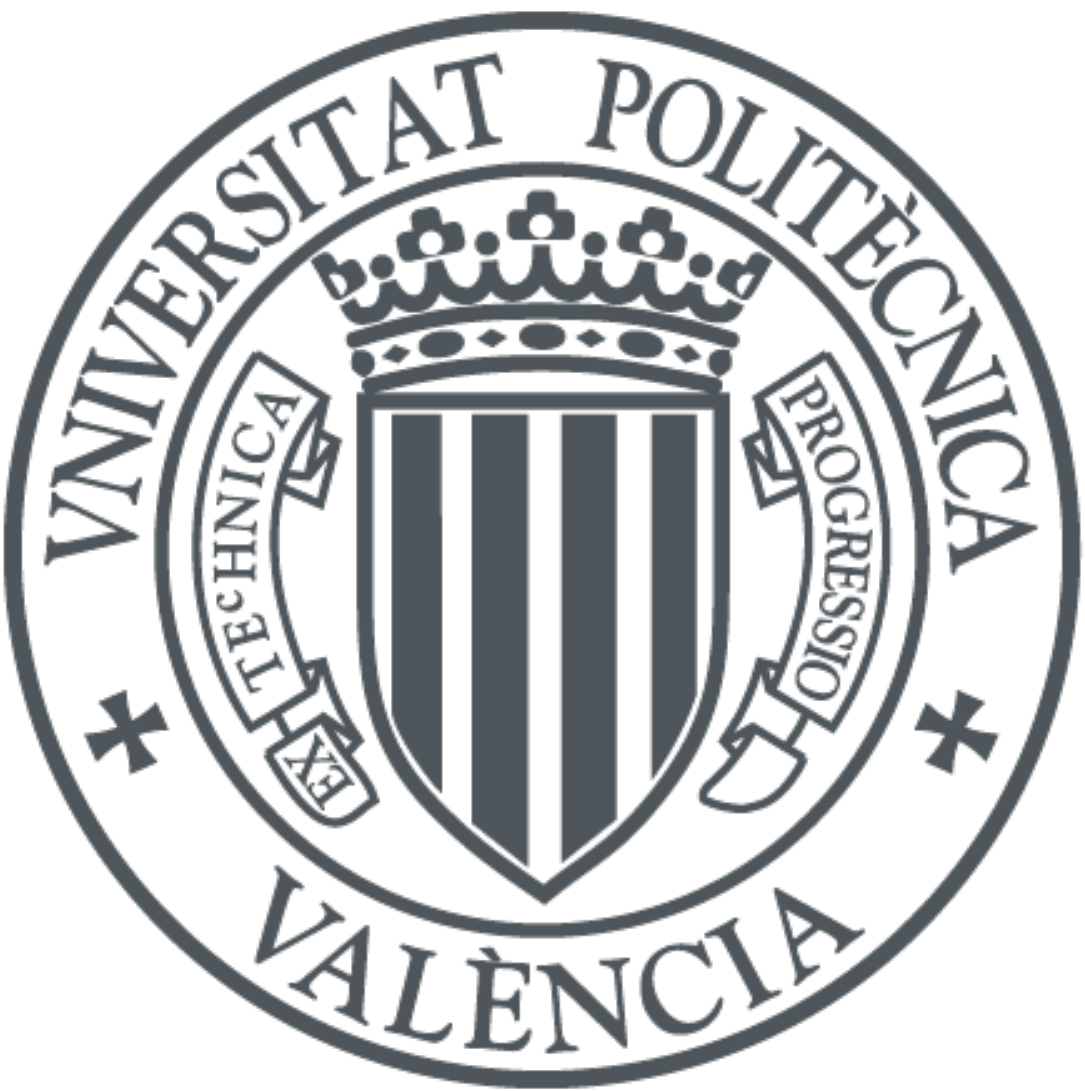

The final publication is available at

https://doi.org/10.1109/EMBC.2019.8857563

Copyright IEEE

Additional Information 


\title{
Distance and Similarity Measurements of $P$ Waves Before and After Pulmonary Vein Isolation in Patients with Atrial Fibrillation
}

\author{
Nuria Ortigosa ${ }^{1}$, Óscar $\mathrm{Cano}^{2}$, Luca Mainardi ${ }^{3}$
}

\begin{abstract}
This study aimed to assess electric markers obtained from the surface electrocardiogram in order to analyse significant differences before and after pulmonary vein isolation in patients who suffered from paroxysmal atrial fibrillation. 30 patients who underwent catheter ablation (in order to permanently restore sinus rhythm and stop atrial fibrillation episodes) were included in the study. Both surface electrocardiogram and intracavitary recordings were simultaneously acquired starting some minutes before catheter ablation began until the whole procedure successfully ended. P-waves have been delineated on $\mathrm{V} 1$ lead, and measurements of distances and similarities between them have been obtained to compare the recordings. It has been found that distances between Pwaves significatively decrease (about $14 \%$ ) whereas similarities significatively increase (about $3 \%$ ) after catheter ablation. The use of these features would help to identify the success of the catheter ablation procedure, which is the main objective of this preliminary study: the non-invasive identification of spontaneous reconnection of pulmonary veins, the main cause of the arrhythmia recurrences.
\end{abstract}

\section{INTRODUCTION}

Atrial Fibrillation (AF) is the most common sustained arrhythmia in clinical practice [1]. Its prevalence grows markedly with age and, due to the increment in global life expectancy, its importance in relation with other arrhythmias will grow up, even nowadays it already affects millions of patients worldwide [2].

Atrial fibrillation classification is based on the duration of the arrhythmic episodes. Patients can be stratified as paroxysmal, if they present self-terminating episodes within 7 days; persistent, if the arrhythmia lasts for more than 7 days; long-standing persistent, if the arrhythmia lasts for more than one year; and permanent, if cardioversion is not successful and sinus rhythm cannot be restored [3].

AF treatment includes antiarrhythmic drugs and non pharmacological treatments, such as catheter ablation of pulmonary veins. The efficacy of each treatment depends on the

N. Ortigosa acknowledges the support from Generalitat Valenciana under grant Prometeo/2017/102; from Spanish Ministerio de Educación, Cultura y Deporte en el marco del Programa Estatal de Promoción del Talento y su Empleabilidad en I+D+i -Subprograma Estatal de Movilidad- under grant José Castillejo CAS18/00396; from Spanish MINECO under grant MTM2016-76647-P, and from VLC-BIOMED 2017 (Universitat de València and Hospital La Fe / IIS La Fe) under grant 10-ARVEAP-GALBIS-CANO2017-A.

${ }^{1}$ Nuria Ortigosa is with I.U. Matemática Pura y Aplicada, Universitat Politècnica de València. Camino de Vera s/n, Edif. 8E, acceso F, 46022 Valencia, Spain nuorar@upvnet.upv.es

${ }^{2}$ Óscar Cano is with Unidad de Arritmias, Servicio de Cardiología, Hospital Universitari i Politècnic La Fe. Av. Fernando Abril Martorell 106, 46026 Valencia, Spain. cano_osc@gva.es

${ }^{3}$ Luca Mainardi is with Department of Electronics, Information and Bioengineering; Politecnico di Milano. Via Ponzio 34/5, 20133 Milan, Italy. luca.mainardi@polimi.it clinical classification of AF [4]. Regarding catheter ablation of pulmonary veins, this technique is able to maintain sinus rhythm in $80 \%$ of patients with paroxysmal atrial fibrillation, but the success rates drop to 40 to $60 \%$ of success of freedom from arrhythmia for persistent patients.

In addition, it is well known that spontaneous electrical reconnection of pulmonary veins is one of the major causes of clinical recurrence of AF after catheter ablation procedures [5], [6]. This spontaneous reconnection has been studied by many references in the last decade [7], [8], [9] but, unfortunately, nowadays there are still no non-invasive markers able to detect this spontaneous reconnection before the patient suffers a new arrhythmia episode again.

Since the identification of predictors of pulmonary vein reconnection would be of great help in clinical practice, in this paper we propose the analysis of different markers extracted from the P-waves of the surface ECG, whose main aim is to confirm the complete isolation of pulmonary veins after catheter ablation procedures.

\section{MATERIALS}

30 patients suffering from paroxysmal atrial fibrillation were included in the study. All of them underwent pulmonary vein isolation in an arrhythmia clinic of a tertiary centre (Hospital Universitari i Politècnic La Fe, Valencia -Spain). Clinical characteristics of the patients are detailed in Table I. The Institution's Ethical Review Board approved all experimental procedures involving human subjects described in this paper.

Surface electrocardiogram and intracavitary recordings were simultaneously acquired by the Labsystem Pro EP Recording System, Boston Scientific. Recordings started some minutes before the beginning of the procedure until catheter ablation was completely and successfully performed.

TABLE I

CLINICAL CHARACTERISTICS OF THE 30 PATIENTS INCLUDED IN THE STUDY. AGE IS DETAILED BY MEAN AND STANDARD DEVIATION, WHEREAS THE REST OF CHARACTERISTICS INDICATE THE NUMBER OF PATIENTS WITH THAT ONE, AND THE PERCENTAGE FROM THE TOTAL.

\begin{tabular}{|c||c|}
\hline Feature & \\
\hline Age & $55 \pm 12$ \\
\hline Male (n, \%) & $20(67 \%)$ \\
\hline High blood pressure & $15(50 \%)$ \\
\hline Diabetes mellitus & $3(10 \%)$ \\
\hline Dyslipidemia & $7(23 \%)$ \\
\hline Left atrial dilation & $20(67 \%)$ \\
\hline
\end{tabular}


Thus, each patient was constantly monitored. As all patients included in the study had paroxysmal AF episodes, we only have extracted the ECG segments corresponding to precordial V1 lead in which each subject was in sinus rhythm. V1 lead was chosen since $\mathrm{P}$ waves can easily be seen on it.

\section{METHODS}

\section{A. Signal preprocessing}

First step was to remove powerline interference by means of a Notch filter at $50 \mathrm{~Hz}$. The ECG signal was also filtered in order to remove baseline wander by using cubic splines [10].

Then, two segments of one-minute length of lead V1 were extracted: the first one corresponding to recordings before catheter ablation began, and another one corresponding to minutes after the procedure had successfully ended. The length of the recordings was set to one minute in order to have enough $\mathrm{P}$-waves in each recording. $\mathrm{P}$ waves were carefully delineated on them, marking their onsets and endings and been revised one by one by an expert cardiologist.

\section{B. Distance measurements}

For each patient, Euclidean distance between each pair of two consecutive P-waves from each recording (before and after catheter ablation) were calculated. Given the matrix $\mathrm{P}$ of size $m \times n$, which contains the $m \mathrm{P}$-waves in the recording, where $n$ is the length in samples of each P-wave, we have obtained a vector of distances $D$.

This vector $D$ contains each one of the Euclidean distances between each pair of two consecutive P-waves $\left(d_{m}\right)$ normalized by the total number of samples $(n)$ for the $m^{\text {th }}$ P-wave $\left(P_{m}\right)$, calculated as:

$$
d_{m}^{2}=\frac{\left(P_{m}-P_{m+1}\right)\left(P_{m}-P_{m+1}\right)^{t}}{n}
$$

where $P_{m}$ denotes the amplitude for the samples of one P-wave in particular, $P_{m+1}$ denotes the amplitude for the samples of the next $\mathrm{P}$-wave in the recording, and $t$ denotes the vector transpose.

Once we have vector $D$, we proceeded to calculate the average value of all its components, obtaining the feature for the analysis.

As each patient had different amplitudes and durations of $\mathrm{P}$-waves, a normalized value of the distance was also calculated, in order to have those values in the interval [0-1]. Thus, we obtained the vector of normalized Euclidean distances $D N$ composed by the different normalized distances $\left(d n_{m}^{2}\right)$ calculated as:

$$
d n_{m}^{2}=\frac{\left(P_{m}-P_{m+1}\right)\left(P_{m}-P_{m+1}\right)^{t}}{\sqrt{\sum_{i=1}^{n} P_{m}^{2}}}
$$

After that, an average of all the components of $D N$ was also obtained and included in the analysis.

\section{Similarity measurements}

A set of measurements to evaluate the similarity between each P-wave and the consecutive one is also obtained $\left(s_{m}\right)$ :

$$
s_{m}=\frac{\left(P_{m} \cdot P_{m+1}^{t}\right)}{\left\|P_{m}\right\| \cdot\left\|P_{m+1}\right\|}
$$

where $t$ denotes the vector transpose and $\left\|P_{m}\right\|$ is the magnitude or Euclidean norm of P-wave $P_{m}$ :

$$
\left\|P_{m}\right\|=\sqrt{\sum_{i=1}^{n}\left|P_{m}\right|^{2}}
$$

An average of all $s_{m}$ values (i.e. similarity measurements calculated for pairs of P-waves of a ECG segment) is obtained and included in the comparison analysis of ECG recordings. Similarity is maximum when it is equal to 1 , and is smaller as long as it decreases towards 0 .

\section{Statistical analysis}

First, Shapiro-Wilk's test was performed to verify if the data followed a normal distribution. As the null hypothesis was rejected for all features, a paired Wilcoxon test was performed in order to compare the data before and after catheter ablation.

\section{RESULTS}

Once P-waves are delineated, different features are obtained to compare similarity and distance differences before and after the procedure. Table II shows median and interquartile range (Q3-Q1) values (also depicted in boxplots of Fig. 1).

It can be observed that the average distances between pairs of P-waves (both with absolute value and normalized) decrease after catheter ablation of pulmonary veins, even only the normalized distance values provide statistically significant differences when comparing the features before/after the procedure using the paired statistical t-test, probably due to the P-wave amplitude differences between patients.

In addition, it can be also observed that similarity measurements between consecutive P-waves also provide statistically significant differences. Similarity is increased after catheter ablation for $70 \%$ of patients, whereas the normalized distance values become smaller for $67 \%$ of patients.

Thus, the disorganized atrial activity present in patients with atrial fibrillation episodes is reduced after the procedure, and it can be related to the significant variations in the features analysed in the present study. A graphical example of how the electric disorganization of atrial activity is reduced after catheter ablation is shown in Fig. 2, where it can be observed that P-waves have less dispersion and are more 'regular' and similar between them after the procedure. This is also reflected on the major similarity of $\mathrm{P}$ wave durations after the catheter ablation procedure. 


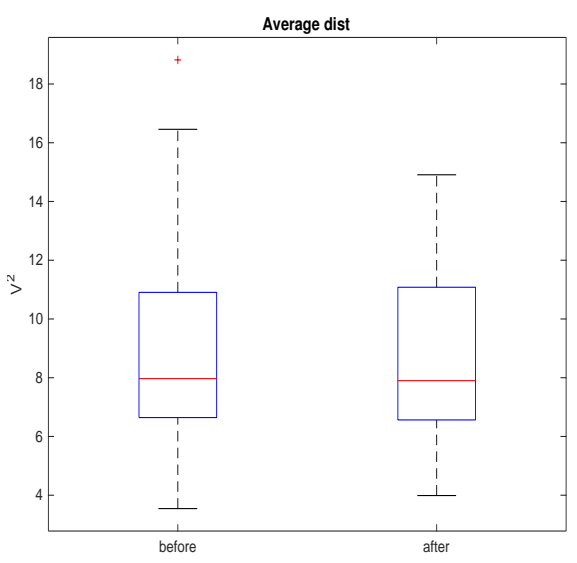

(a)

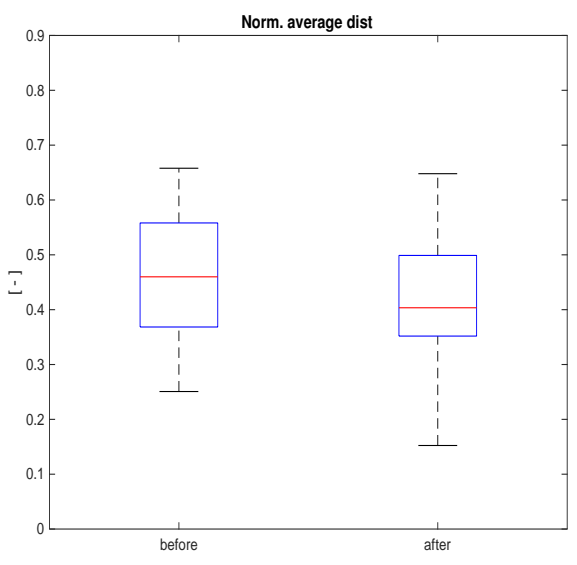

(b)

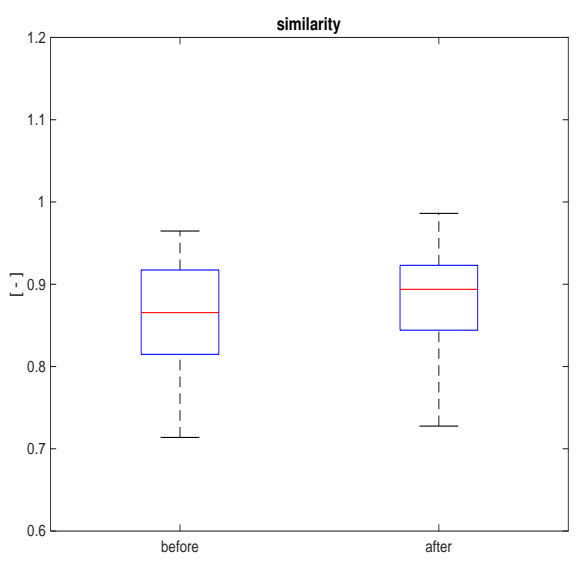

(c)

Fig. 1. Boxplot of features obtained before and after catheter ablation procedure on the cohort under study. (a) Average distance, (b) Normalized average distance, and (c) Similarity.

TABLE II

MEdIAN AND INTERQUARTILE RANGE OF FEATURES OBTAINED FOR THE

30 SUBJECTS INCLUDED IN THE STUDY. RESULTS SHOW VALUES

CALCULATED ON ECG P-WAVES BEFORE AND AFTER CATHETER ABLATION. SIGNIFICANT DIFFERENCES ARE INDICATED BY * (P-VALUE $<0.05)$ AFTER THE CALCULATION OF PAIRED WILCOXON TEST.

\begin{tabular}{|c||c|c|c|}
\hline Feature & Before & After & p-value \\
\hline Similarity $[-]$ & $0.866(0.815-0.917)$ & $0.894(0.844-0.923)$ & $0.007^{*}$ \\
\hline Distance $\left[V^{2}\right]$ & $7.970(6.644-10.906)$ & $7.901(6.563-11.080)$ & 0.357 \\
\hline $\begin{array}{c}\text { Normalized } \\
\text { Distance }[-]\end{array}$ & $0.459(0.368-0.558)$ & $0.403(0.351-0.499)$ & $0.011^{*}$ \\
\hline
\end{tabular}

\section{DISCUSSION}

Presented results go in line with previous studies [11], [12]. Patients with AF are characterized by the disorganization of atrial activity. The reduction of the distances and the increment of similarities between consecutive pairs of P-waves after catheter ablation of pulmonary veins may be explained by the reduction of disorganized propagation of electric activity through the atria, which is the main aim of catheter ablation to permanently restore sinus rhythm in AF patients [13].

These differences may be due to the reduction of the fragmentation and the atrial activity disorganization of patients after the procedure. The use of features extracted from recordings acquired by non-invasive methods, such as the surface ECG, may open the door to help clinicians in the follow-up of patients and early detect possible recurrences of the arrythmia.

Future work will focus on enlarge the population under study and analyse the future recurrences of the patients involved in the study, in order to study which features can be considered as non-invasive predictors of spontaneous pulmonary veins reconnection.

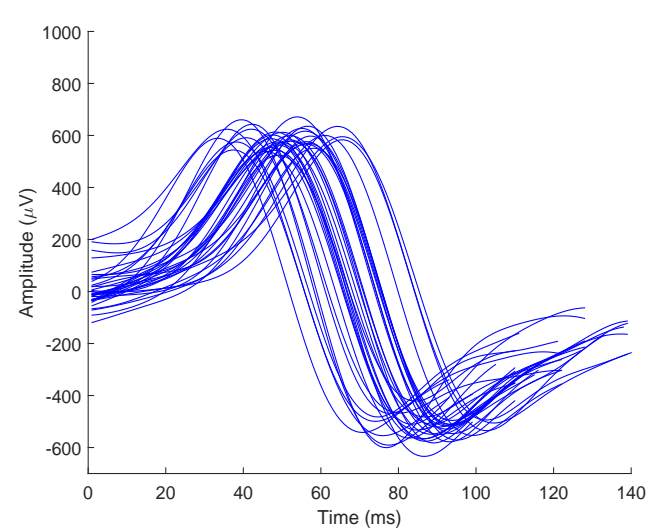

(a)

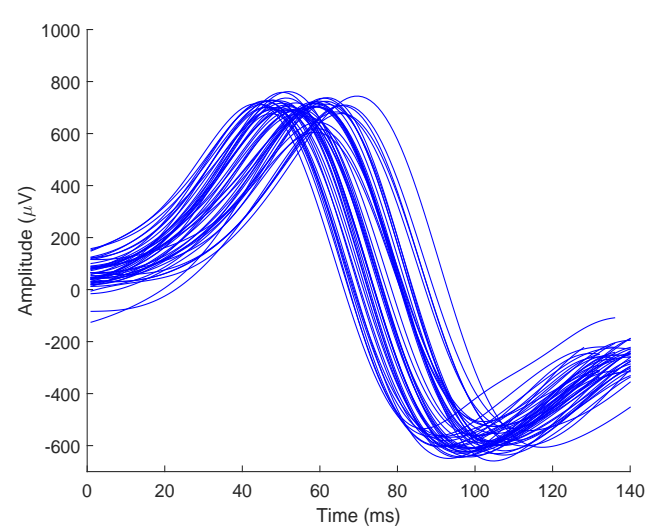

(b)

Fig. 2. Example of set of P-waves extracted from V1 lead of a patient with paroxysmal AF. (a) Before catheter ablation beginning. (b) After complete and successful catheter ablation is performed.

\section{CONCLUSIONS}

In this paper we have presented a study whose aim is to analyse the differences in the surface electrocardiogram that can be found before and after pulmonary vein isolation in patients with paroxysmal atrial fibrillation. 
It has been shown that the average of normalized Euclidean distances between consecutive pairs of P-waves statistically significantly decrease when sinus rhythm is permanently restored, as well as the average of similarities calculated over pairs of consecutive P-waves is incremented.

\section{REFERENCES}

[1] L. Wann, A. Curtis, C. January, K. Ellenbogen, J. Lowe, and N. E. et al., "2011 ACCF/AHA/HRS focused update on the management of patients with atrial fibrillation (updating the 2006 guideline): a report of the American College of Cardiology Foundation/American Heart Association Task Force on Practice Guidelines," Circulation, vol. 123, no. 1, pp. 104-123, 2011.

[2] P. Platonov and V. Corino, Atrial Fibrillation from an Engineering Perspective. Springer, 2018, ch. A Clinical Perspective on Atrial Fibrillation.

[3] C. January, L. Wann, J. Alpert, H. Calkins, J. Cigarroa, and J. C. et al., "2014 AHA/ACC/HRS Guideline for the Management of Patients With Atrial Fibrillation. A Report of the American College of Cardiology/American Heart Association Task Force on Practice Guidelines and the Heart Rhythm Society," J. Am. Coll. Cardiol., vol. 64, no. 21, pp. e1-e76, December 2014.

[4] J. Melichercik, "New frontiers in the evaluation and treatment of patients with atrial fibrillation," Biomed. Tech., vol. 57, no. 1, 2012.

[5] E. Anter, F. Contreras-Valdes, A. Shvilkin, C. Tschabrunn, and M. Josephson, "Acute pulmonary vein reconnection is a predictor of atrial fibrillation recurrence following pulmonary vein isolation," $J$ Interv Card Electrophysiol., vol. 39, no. 3, pp. 225-232, April 2014.

[6] M. Efremidis, L. Letsas, G. Giannopoulos, L. Lioni, K. Vlachos, D. Asvestas, D. Karlis, V. Kareliotis, H. Geladari, and A. S. et al., "Early pulmonary vein reconnection as a predictor of left atrial ablation outcomes for paroxysmal atrial fibrillation," Europace, vol. 17, no. 5, pp. 741-746, May 2015.

[7] G. Epicoco and A. Sorgente, "Predictors of Atrial Fibrillation Recurrence after Catheter Ablation," J Atr Fibrillation, vol. 6, no. 5, p. 1016, February-March 2014.

[8] C.H. Calkins and K.H. Kuck and R. Cappato and J. Brugada and A.J. Camm, et al., "2012 HRS/EHRA/ECAS expert consensus statement on catheter and surgical ablation of atrial fibrillation: Recommendations for patient selection, procedural techniques, patient management and follow-up, definitions, end- points, and research trial design," J Interv Card Electrophysiol, vol. 33, no. 2, pp. 171-257, 2012.

[9] A. Ganesan, N. Shipp, A. Brooks, P. Kuklik, and D. L. et al., "Longterm outcomes of catheter ablation of atrial fibrillation: A systematic review and met-analysis," J Am Heart Assoc, vol. 2, no. 2, p. e004549, 2013.

[10] C. R. Meyer and H. N. Keiser, "Electrocardiogram baseline noise estimation and removal using cubic splines and state-space computation techniques," Comput. Biomed. Res., vol. 10, pp. 459-470, 1977.

[11] N. Ortigosa and O. Cano, "P-wave Analysis in Paroxysmal Atrial Fibrillation Patients before and after Pulmonary Vein Isolation," Computing in Cardiology, vol. 44, no. 6, pp. 1-4. DOI:10.22 489/CinC.2017.284-091, 2017.

[12] F. Censi, I. Corazza, E. Reggiani, G. Calcagnini, E. Mattei, M. Triventi, and G. Boriani, "P-wave Variability and Atrial Fibrillation," Scientific Reports, vol. 6, pp. 1-7, May 2016.

[13] T. McGarry and S. Narayan, "The Anatomical Basis of Pulmonary Vein Reconnection After Ablation for Atrial Fibrillation," J Am Coll Cardiol., vol. 59, no. 10, pp. 939-941, March 2012. 